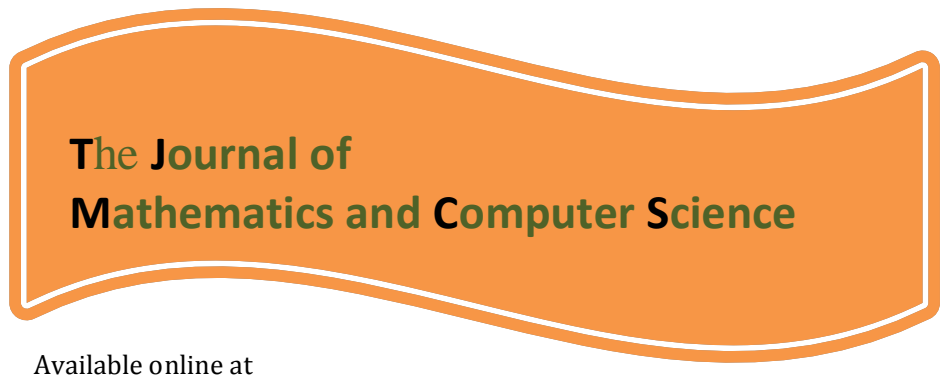

http://www.TJMCS.com

The Journal of Mathematics and Computer Science Vol .2 No.4 (2011) 588-593

\title{
Closedness of the rang of the product of projections in Hilbert modules
}

\author{
Kamran Sharifi \\ Department of Mathematics, Shahrood University of Technology, \\ P. 0. Box 3619995161-316, Shahrood, Iran \\ sharifi.kamran@gmail.com \& sharifi@shahroodut.ac.ir
}

Received: July 2010, Revised: October 2010

Online Publication: January 2011

\begin{abstract}
.
Suppose $P$ and $Q$ are orthogonal projections between Hilbert $\mathrm{C}^{*}$-modules, then $P Q$ has closed range if and only if $\operatorname{Ker}(P)+\operatorname{Ran}(Q)$ is an orthogonal summand, $\operatorname{Ker}(Q)+\operatorname{Ran}(P)$ is an orthogonal summand.
\end{abstract}

Keywords: Hilbert $C^{*}$-module, projection, Moore-Penrose inverse, closed range.

AMS subject classifications 46L08, 47A05, 15A09, 46L05

\section{Introduction and preliminaries.}

The closedness of range of operators is an attractive and important problem which appears in operator theory, especially, in the theory of Fredholm operators and Moore-Penrose inverses. In this paper we will investigate when the product of two orthogonal projections has closed range. This problem was first studied by Bouldin for bounded operators between Hilbert spaces in [1, 2], and then was reconsidered by authors in $[3,7,9]$.

A Hilbert $C^{*}$-module obeys the same axioms as an ordinary Hilbert space, 
except that the inner product, from which the geometry emerges, takes values in an arbitrary $\mathrm{C}^{*}$-algebra A rather than $\mathrm{C}$. Some well-known and fundamental properties of Hilbert spaces like Pythagoras' equality, self-duality, and even decomposition into orthogonal complements must be given up in these modules. They play an important role in the modern theory of $\mathrm{C}^{*}$-algebra, notably, in noncommutative geometry and in quantum groups, see [6].

A (left) pre-Hilbert $C^{*}$-module over a (not necessarily unital) $\mathrm{C}^{*}$-algebra $\mathrm{A}$ is a left $\mathrm{A}$-module $X$ equipped with an $\mathrm{A}$-valued inner product $\langle\cdot, \cdot\rangle: X \times X \rightarrow \mathrm{A}$, which is $\mathrm{A}$-linear in the first variable and has the properties:

$$
\langle x, y\rangle=\langle y, x\rangle^{*}, \quad\langle x, x\rangle \geq 0 \text { withequality if and onlyif } x=0 .
$$

We always suppose that the linear structures of $A$ and $X$ are compatible. A preHilbert $\mathrm{A}$-module $X$ is called a Hilbert $\mathrm{A}$-module if $X$ is a Banach space with respect to the norm $\|x\|=\|\langle x, x\rangle\|_{\mathrm{A}}^{1 / 2}$. A Hilbert $\mathrm{A}$-submodule $X$ of a Hilbert $\mathrm{A}$ module $Y$ is an orthogonal summand if $Y=X \oplus X^{\perp}$, where $X^{\perp}:=\{y \in Y:\langle x, y\rangle=0$ for all $x \in X\}$ denotes the orthogonal complement of $X$ in $Y$. The book [10] is used as a standard source of reference.

Throughout the present paper we assume $\mathrm{A}$ to be an arbitrary $\mathrm{C}^{*}$-algebra (i.e. not necessarily unital). We use the notations $\operatorname{Ker}($.$) and \operatorname{Ran}($.$) for kernel and$ range of operators, respectively. We denote by $\mathrm{L}(X)$ the $\mathrm{C}^{*}$-algebra of all bounded adjointable operators on $X$, i.e., all bounded A -linear maps $T: X \rightarrow X$ such that there exists $T^{*}: X \rightarrow X$ with the property $\langle T x, y\rangle=\left\langle x, T^{*} y\right\rangle$ for all $x, y \in X$.

In this paper we first briefly investigate some basic facts about closed range operators and Moore-Penrose inverses of bounded adjointable operators on Hilbert $\mathrm{C}^{*}$-modules, then we give some necessary and sufficient conditions for closedness of the range of the product of two orthogonal projections.

Closed submodules of Hilbert modules need not to be orthogonally complemented at all, but Lance states in [10, Theorem 3.2] under which conditions closed submodules may be orthogonally complemented. Let $X$ be a Hilbert A module and suppose that an operator $T$ in $\mathrm{L}(X)$ has closed range, then one has:

- $\operatorname{Ker}(T)$ is orthogonally complemented in $X$, with complement $\operatorname{Ran}\left(T^{*}\right)$,

- $\operatorname{Ran}(T)$ is orthogonally complemented in $X$, with complement $\operatorname{Ker}\left(T^{*}\right)$,

- the map $T^{*} \in \mathrm{L}(X)$ has closed range, too.

The result are extended to unbounded adjointable operators between Hilbert $\mathrm{C}^{*}$-modules in [4].

Lemma 1.1 Suppose $T$ in $\mathrm{L}(X)$ is a bounded adjointable operator. The operator $T$ has closed range if and only if $T T^{*}$ has closed rang. In this case, $\operatorname{Ran}(T)=\operatorname{Ran}\left(T T^{*}\right)$.

Proof. Suppose $T$ has closed range, the proof of Theorem 3.2 of [10] indicates that $\operatorname{Ran}\left(T T^{*}\right)$ is closed and $\operatorname{Ran}(T)=\operatorname{Ran}\left(T T^{*}\right)$.

Conversely, if $T T^{*}$ has closed range then 
$X=\operatorname{Ran}\left(T T^{*}\right) \oplus \operatorname{Ker}\left(T T^{*}\right)=\operatorname{Ran}\left(T T^{*}\right) \oplus \operatorname{Ker}\left(T^{*}\right) \subset \operatorname{Ran}(T) \oplus \operatorname{Ker}\left(T^{*}\right) \subset X \quad$ which implies $T$ has closed range.

Let $T \in \mathrm{L}(X)$, then a bounded adjointable operator $T^{\dagger} \in \mathrm{L}(X)$ is called the Moore-Penrose inverse of $T$ if

$$
T T^{\dagger} T=T, T^{\dagger} T T^{\dagger}=T^{\dagger},\left(T T^{\dagger}\right)^{*}=T T^{\dagger} \text { and }\left(T^{\dagger} T\right)^{*}=T^{\dagger} T \text {. }
$$

The notation $T^{\dagger}$ is reserved to denote the Moore-Penrose inverse of $T$. These properties imply that $T^{\dagger}$ is unique and $T^{\dagger} T$ and $T T^{\dagger}$ are orthogonal projections. Moreover, $\quad \operatorname{Ran}\left(T^{\dagger}\right)=\operatorname{Ran}\left(T^{\dagger} T\right), \quad \operatorname{Ran}(T)=\operatorname{Ran}\left(T T^{\dagger}\right), \quad \operatorname{Ker}(T)=\operatorname{Ker}\left(T^{\dagger} T\right) \quad$ and $\operatorname{Ker}\left(T^{\dagger}\right)=\operatorname{Ker}\left(T T^{\dagger}\right)$ which lead us to $X=\operatorname{Ker}\left(T^{\dagger} T\right) \oplus \operatorname{Ran}\left(T^{\dagger} T\right)=\operatorname{Ker}(T) \oplus \operatorname{Ran}\left(T^{\dagger}\right)$ and $X=\operatorname{Ker}\left(T^{\dagger}\right) \oplus \operatorname{Ran}(T)$.

$\mathrm{Xu}$ and Sheng in [15] have shown that a bounded adjointable operator between two Hilbert $\mathrm{C}^{*}$-modules admits a bounded Moore-Penrose inverse if and only if the operator has closed range. The reader should be aware of the fact that a bounded adjointable operator may admit an unbounded operator as its MoorePenrose, see $[5,13,14]$ for more detailed information.

\section{The product of projections}

Suppose $X$ is a Hilbert $A$-module and $T$ be a bounded adjointable operator in the unital $\mathrm{C}^{*}$-algebra $\mathrm{L}(X)$, then $\sigma(T)$ and acc $\sigma(T)$ denote the spectrum and the set of all accumulation points of $\sigma(T)$, respectively. According to [8, Theorem 2.4] and [15, Theorem 2.2], a bounded adjointable operator $T$ in $\mathrm{L}(X)$ has closed range if and only if $T$ has a Moore-Penrose inverse, if and only if $0 \notin \mathrm{acc} \sigma\left(T T^{*}\right)$, if and only if $0 \notin \mathrm{acc} \sigma\left(T^{*} T\right)$. In particular, when $T$ is selfadjoint the above facts read as follows:

Lemma 2.1 Suppose $X$ is a Hilbert A -module and $T \in \mathrm{L}(X)$ is selfadjoint then $T$ has closed range if and only if $0 \notin \mathrm{acc} \sigma(T)$.

Proof. Suppose $T$ is a selfadjoint element of the $C^{*}$-algebra $\mathrm{L}(X)$, by the Spectral Mapping Theorem we have $\sigma\left(T^{2}\right)=\sigma(T)^{2}$. The later equality and the above facts imply that $T$ has closed range if and only if $0 \notin \mathrm{acc} \sigma(T)$.

Lemma 2.2 Suppose $X$ is a Hilbert A -module and $P, Q$ are orthogonal projections in $\mathrm{L}(X)$. Then $P-Q$ has closed range if and only if $P+Q$ has closed range.

Proof. Following the argument of Koliha and Rako čevi c' [9], for every $\lambda \in \mathrm{C}$ we have

$$
\begin{aligned}
(\lambda-1+P)(\lambda-(P-Q))(\lambda+1-Q) & =((\lambda-1)(\lambda+Q)+P Q)(\lambda+1-Q) \\
& =\lambda\left(\lambda^{2}-1+P Q\right),
\end{aligned}
$$




$$
\begin{aligned}
(\lambda-1+P)(\lambda-(P+Q))(\lambda-1+Q) & =((\lambda-1)(\lambda-Q)-P Q)(\lambda-1+Q) \\
& =\lambda\left((\lambda-1)^{2}-P Q\right) .
\end{aligned}
$$

Using the above equations and the facts that $\sigma(P) \subset\{0,1\}$ and $\sigma(Q) \subset\{0,1\}$, we obtain that $\operatorname{Ran}(P-Q)$ is closed if and only if $0 \notin \mathrm{acc} \sigma(P-Q)$, if and only if $1 \notin \mathrm{acc} \sigma(P Q)$, if and only if $0 \notin \mathrm{acc} \sigma(P+Q)$, if and only if $\operatorname{Ran}(P+Q)$ is closed.

Lemma 2.3 (cf. [11, Remark 1.2.1]) If $a$ and $b$ are elements of a unital $C^{*}$-algebra A , then $\sigma(a b) \backslash\{0\}=\sigma(b a) \backslash\{0\}$.

Lemma 2.4 Suppose $X$ is a Hilbert A-module and $P, Q$ are orthogonal projections in $\mathrm{L}(X)$. Then the following conditions are equivalent:

- $P Q$ has closed range,

- $1-P-Q$ has closed range,

- $1-P+Q$ has closed range,

- $1-Q+P$ has closed range.

Proof. Suppose $\lambda \in \mathrm{C} \backslash\{0,1\}$. In view of the equation (3), we conclude that $\lambda \in \sigma(P+Q)$ if and only if $(\lambda-1)^{2} \in \sigma(P Q)$.

The above fact together with Lemma 2.3 imply that $P Q$ has closed range if and only if $0 \notin \mathrm{acc} \sigma(P Q P)$, if and only if $0 \notin \mathrm{acc} \sigma\left(P^{2} Q\right)=\mathrm{acc} \sigma(P Q)$, if and only if $1 \notin$ acc $\sigma(P+Q)$, if and only if $0 \notin$ acc $\sigma(1-P-Q)$, if and only if $1-P-Q$ has closed range. This proves the equivalence of (i) and (ii). The statements (ii), (iii) and (iv) are equivalent by Lemma 2.2 .

Remark 2.5 Suppose $X, Y$ are two Hilbert A-modules then the set of all ordered pairs of elements $X \oplus Y$ from $X$ and $Y$ is a Hilbert $A$-module with respect to the A valued inner product $\left\langle\left(x_{1}, y_{1}\right),\left(x_{2}, y_{2}\right)\right\rangle=\left\langle x_{1}, x_{2}\right\rangle_{X}+\left\langle y_{1}, y_{2}\right\rangle_{Y}$, cf. [12, Example 2.14]. In particular, it can be easily seen that $W$ is a closed submodule of $X$ if and only if $W \oplus\{0\}$ is a closed submodule of $X \oplus X$.

Theorem 2.6 Suppose $P$ and $Q$ are orthogonal projections on a Hilbert A -module $X$ then the following conditions are equivalent:

- $P Q$ has closed range,

- $\operatorname{Ker}(P)+\operatorname{Ran}(Q)$ is an orthogonal summand,

- $\operatorname{Ker}(Q)+\operatorname{Ran}(P)$ is an orthogonal summand.

Proof. Suppose

$$
T=\left(\begin{array}{cc}
1-P & Q \\
0 & 0
\end{array}\right) \in \mathrm{L}(X \oplus X)
$$


Then $\quad \operatorname{Ran}(T)=(\operatorname{Ran}(1-P)+\operatorname{Ran}(Q)) \oplus\{0\} \quad$ and $\quad \operatorname{Ran}\left(T T^{*}\right)=\operatorname{Ran}(1-P+Q) \oplus\{0\}$. Using Lemmata 1.1, 2.4 and Remark 2.5, we infer that $P Q$ has closed range if and only if $1-P+Q$ has closed range, if and only if $\operatorname{Ran}\left(T T^{*}\right)=\operatorname{Ran}(1-P+Q) \oplus\{0\}$ is closed, if and only if $\operatorname{Ran}(T)=(\operatorname{Ran}(1-P)+\operatorname{Ran}(Q)) \oplus\{0\}$ is closed, if and only if $\operatorname{Ran}(1-P)+\operatorname{Ran}(Q)$ is closed. In this case, $\operatorname{Ran}\left(T T^{*}\right)=\operatorname{Ran}(T)$ which implies that $\operatorname{Ran}(1-P+Q)=\operatorname{Ran}(1-P)+\operatorname{Ran}(Q)$ is an orthogonal summand. These prove that the conditions (i) and (ii) are equivalent. Now, consider the matrix operator

$$
\tilde{T}=\left(\begin{array}{cc}
1-Q & P \\
0 & 0
\end{array}\right) \in \mathrm{L}(X \oplus X) .
$$

A similar argument show that $P Q$ has closed range if and only if $\operatorname{Ran}(1-Q+P)=\operatorname{Ran}(1-Q)+\operatorname{Ran}(P)$ is closed which shows that conditions (i) and (iii) are equivalent.

Acknowledgement: This research was supported by a grant of Shahrood University of Technology.

\section{References}

[1] R. Bouldin, The product of operators with closed range, Tôhoku Math. J. (2) 25 (1973), 359-363.

[2] R. Bouldin, Closed range and relative regularity for products, J. Math. Anal. Appl. 61 (1977), 397-403.

[3] F. Deutsch, The angle between subspaces in Hilbert space, Approximation theory, wavelets and applications (S. P. Singh, editor), Kluwer, Netherlands, 1995, 107-130.

[4] M. Frank and K. Sharifi, Adjointability of densely defined closed operators and the Magajna-Schweizer Theorem, J. Operator Theory 63 (2010), 271-282.

[5] M. Frank and K. Sharifi, Generalized inverses and polar decomposition of unbounded regular operators on Hilbert $\mathrm{C}^{*}$-modules, to appear in J. Operator Theory. available on arXiv:math.0A/0806.0162 v1 1 Jun 2008.

[6] J. M. Gracia-Bonda, J. C. Várilly, H. Figueroa, Elements of noncommutative geometry, Birkhäuser, 2000.

[7] S. Izumino, The product of operators with closed range and an extension of the revers order law, Tôhoku Math. J. (2) 34 (1982), 43-52.

[8] J. J. Koliha, The Drazin and Moore-Penrose inverse in $\mathrm{C}^{*}$-algebras, Proc. Roy. Irish Acad. Sect. A 99 (1999), 17-27.

[9] J. J. Koliha and V. Rako č evi c' , Fredholm properties of the difference of orthogonal projections in a Hilbert space, Integr. equ. oper. theory 52 (2005), no. 1, 125-134.

[10] E. C. Lance, Hilbert C*-Modules, LMS Lecture Note Series 210, Cambridge Univ. Press, 1995.

[11] G. J. Murphy, $C^{*}$-algebras and Operator Theory, Academic Press, 1990. 
[12] I. Raeburn and D. P. Williams, Morita Equivalence and Continuous Trace $C^{*}$-algebras, Math. Surveys and Monogr. v. 60, Amer. Math. Soc., Providence, R. I., 1998.

[13] K. Sharifi, Descriptions of partial isometries on Hilbert C*-modules, Linear Algebra Appl. 431 (2009), 883-887.

[14] K. Sharifi, Groetsch's Representation of Moore-Penrose inverses and illposed problems in Hilbert C*-modules, J. Math. Anal. Appl. 365 (2010), 646-652.

[15] Q. Xu and L. Sheng, Positive semi-definite matrices of adjointable operators on Hilbert C*-modules, Linear Algebra Appl. 428 (2008), 992-1000. 